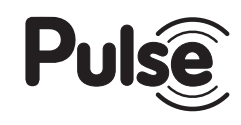

\title{
Logical Management of Aortic Coarctation
}

\author{
Michael F. O'Rourke \\ St Vincent's Clinic, University of New South Wales/VCCRI, Sydney, NSW, Australia
}

Murakami [1] reports his experience with patients undergoing corrective surgery or balloon angioplasty for aortic coarctation. All pressure wave data were collected after treatment, and results are assumed to be a consequence of treatment. This is not the usual interpretation $[2,3]$.

In aortic coarctation, there is a congenital narrowing of the proximal descending thoracic aorta. This is usually sufficiently severe to create an obvious pressure gradient between upper body and lower body arteries. Surgical treatment usually lowers this gradient substantially, but even if the mean gradient is reduced to less than $5 \mathrm{~mm} \mathrm{Hg}$, pulsatile pressure usually remains high in the upper body and late complications of cardiac failure or stroke are more common than in persons without coarctation, though more common in patients where coarctation is not fully corrected by surgery or balloon dilation. The abnormality at the site of the condition causes early wave reflection to the heart and cerebral arteries. As described by Celermajer and Greaves [3], the coarctation is "fixed" but not "cured." Persistence of high augmentation in the proximal thoracic aorta after surgery may warrant repeat of surgical correction at an older age, with use of a stent or graft to minimise obstruction to blood flow. Complete restoration to normality at the site of previous coarctation is not usually possible, so that high pulsatile pressure in the central arteries usually persists throughout life even if the mean pressure gradient appears to have been abolished. The findings of Murakami [1] are not a consequence of surgery and may warrant repeat of surgery at a later date. Patients with corrected coarctation are at high risk and warrant close follow-up. Persisting hypertension may warrant antihypertensive therapy. The optimal drug therapy may be with nitrate-like compounds which dilate large artery collateral vessels. 
O’Rourke: Logical Management of Aortic Coarctation

\section{Disclosure Statement}

Dr O'Rourke is a founding director of AtCor Medical P/L, manufacturer of pulse wave analysis system, SphygmoCor, and of Aortamate $\mathrm{P} / \mathrm{L}$, developer of methods to reduce aortic stiffness.

\section{References}

1 Murakami: Enhanced aortic pressure wave reflection in patients with aortic coarctation after aortic arch repair. Pulse 2017, DOI: 10.1159/000478530.

-2 O'Rourke MF, Cartmill TB: Influence of aortic coarctation on pulsatile hemodynamics in the proximal aorta. Circulation 1971;44:281-292.

-3 Celermajer DS, Greaves K: Survivors of coarctation repair: fixed but not cured. Heart 2002;88:113-114. 